\title{
Réflexions autour de la méthode dite de la sémiotique situationnelle fondées sur une recherche en communication des organisations
}

\section{Barbara Szafrajzen}

\section{(Q) OpenEdition}

\section{Journals}

Édition électronique

URL : http://journals.openedition.org/communicationorganisation/3130

DOI : 10.4000/communicationorganisation.3130

ISSN : $1775-3546$

Éditeur

Presses universitaires de Bordeaux

Édition imprimée

Date de publication : 1 juin 2011

Pagination : 183-194

ISBN : 978-2-86781-744-1

ISSN : $1168-5549$

\section{Référence électronique}

Barbara Szafrajzen, «Réflexions autour de la méthode dite de la sémiotique situationnelle fondées sur une recherche en communication des organisations », Communication et organisation [En ligne], 39 |

2011, mis en ligne le 01 juin 2014, consulté le 10 décembre 2020. URL : http://

journals.openedition.org/communicationorganisation/3130; DOI : https://doi.org/10.4000/ communicationorganisation.3130 


\title{
Réflexions autour de la méthode dite de la sémiotique situationnelle fondées sur une recherche en communication des organisations
}

\author{
Borbora Szafrajzen'
}

Depuis plusieurs années, de nombreuses recherches en sciences de l'information et de la communication (SIC) choisissent de s'intéresser aux pratiques sémiotiques en communication des organisations, tant sur le plan épistémologique, théorique, ou encore méthodologique. Les modèles fondateurs des sémiotiques linguistiques classiques laissent peu à peu place à des applications diverses, permettant aux chercheurs, tout comme aux professionnels, d'avoir recours à la sémiotique comme outil, comme méthode, ou encore comme univers théorique (Boutaud, 2007). La communication organisationnelle est un des domaines des sciences de l'information et de la communication à se nourrir de ces recherches en sémiotique-sémiologie. Parmi les méthodologies possibles de cette relation, nous choisissons de nous intéresser à la méthode d'analyse dite de la sémiotique situationnelle, proposée par Alex Mucchielli au début des années 2000 (Mucchielli, 2003).

Nous nous demandons quels sont les usages, les apports et les limites de cette méthode d'analyse dans les recherches sur les communications organisationnelles ? Nous avons réfléchi à cette question par l'entremise d'une recherche réalisée au sein d'une cité universitaire de la ville de Montpellier (Hérault, France) ${ }^{2}$. Le projet de cette recherche a été de comprendre et d'analyser le fonctionnement de l'organisation citée, de sorte à proposer des préconisations d'intervention auprès des acteurs de l'organisation.

Nous commençons par exposer l'ancrage théorique et conceptuel de la méthode dite de la sémiotique situationnelle. Dans un second temps, nous faisons référence à la recherche menée en SIC et mettant en œuvre la méthode.

1. LERASS (Laboratoire d'études et de recherches appliquées en sciences sociales-EA 827) - Equipe Organicom. szafraizenb@voila.fr

2. Recherche réalisée dans le cadre de notre Master en Sciences de l'Information et de la Communication (mémoire intitulé : " Analyse communicationnelle d'une cité universitaire à Montpellier) durant l'année universitaire 2002-2003 (Université Paul Valéry, Montpellier III). 
Dans un dernier temps, nous mettons en perspective les résultats obtenus de sorte à tenter de contribuer à une réflexion mettant en tension les champs de la sémiotique à la communication des organisations.

\section{I - Ancrage théorique et conceptuel de la méthode}

Un temps appelée "théorie des processus de la communication», ou encore "théorie sémio-contextuelle ", Alex Mucchielli a proposé la méthode d'analyse -aujourd'hui dite de la "sémiotique situationnelle »- alors qu'il était à la direction du laboratoire du CERIC (Centre d'Études et de Recherche en Information et Communication, Montpellier 3).

Cette méthode d'analyse des phénomènes communicationnels puise ses fondements théoriques dans l'approche compréhensive des phénomènes (et plus précisément la sociologie compréhensive), l'ethnométhodologie, la phénoménologie ainsi que l'approche interactionniste et située.

La méthode se propose d'analyser chaque phénomène communicationnel en s'attachant aux points de vue des acteurs en situation (Weber, Parsons, Schütz, 2007). L'auteur convoque le terme « sémiotique » pour comprendre comment un acteur, en observant un phénomène, fait émerger le sens en situation. Il s'agit d'un travail de recontextualisation, en s'intéressant plus précisément aux « communications généralisées significatives " (Mucchielli, 2004) des acteurs (la problématique est celle de la genèse du sens partagé). L'acteur comprend un phénomène et lui donne une signification en mettant en relation ledit phénomène avec d'autres éléments de la situation. L'émergence de ces significations ne peut se faire qu' " en situation en train de se faire " et est liée à l'utilisation de l'interactivité comme moyen d'émergence (LeleuMerviel, 2004). L'ensemble des actions des acteurs sociaux sont replacées au cœur du phénomène à traiter. De ce fait, une action sera toujours replacée dans son contexte (et considérée "en situation ») et analysée en interaction et au regard des enjeux, des positionnements, des normes, inhérents aux acteurs en présence ${ }^{3}$. C'est en ce sens que toute action est considérée comme contextualisée dans la mesure où chaque acteur sélectionne (en fonction de ses préoccupations et centres d'intérêts) des éléments pertinents de son environnement, faisant sens pour lui. Ce processus dynamique met en jeu des contextes spécifiques, construisant et reconstruisant le sens en situation.

En observant comment les acteurs agissent en situation à travers la réalisation de communications processus ${ }^{4}$, l'analyste comprend comment la situation prend un sens spécifique pour l'acteur. Ces communications processus interviennent sur différents contextes de la situation et font émerger la construction du sens de la situation pour l'acteur concerné.

3. Cela sous-entend également que l'on n'envisage pas d'analyser un fait humain sans prendre en considération les usagers, et par là-même leurs usages, autrement dit leurs activités humaines.

4. Alex Mucchielli parle de communication processus pour définir les expressions verbales (ou non verbales) ainsi que les conduites et activités humaines. 
Sachant que les acteurs d'une même organisation ne partagent pas toujours la même vision ou définition d'un même phénomène, la méthode de la sémiotique situationnelle a pour objectif de mettre en exergue ces contextes de sorte à reconstruire et faire émerger le sens final de la communication. Ces divergences de points de vue viennent tant des places et rôles tenus que d'autres facteurs comme l'éducation, le vécu, l'engagement, les enjeux. Sachant que l'action d'un acteur est largement influencée par la représentation qu'il se fait de la situation (Bouzon, 2010), le recours à cette méthode doit permettre à l'ensemble des acteurs de l'organisation de sortir de leur vision personnelle et individuelle de la situation de façon à prendre de la distance sur les interprétations et le sens qu'ils en donnent. En prenant de la hauteur sur la situation, l'acteur peut élargir son champ de vision et percevoir une situation bien plus globale, et bien plus complexe. C'est en présentant cette vision panoramique regroupant l'ensemble des points de vue de tous les acteurs qu'il devient possible de trouver des solutions pour parvenir à une vision commune, connue et partagée par tous les acteurs impliqués dans la situation. Pour ce faire, la méthode d'analyse se propose d'intervenir sur deux grands types de situations-problèmes : les situations au sein desquelles les acteurs ne parviennent pas à l'intercompréhension (malgré leurs échanges), et les situations où les acteurs manipulent des contextes pertinents pour d'autres acteurs, afin de faire changer d'attitude ou de comportement.

C'est en ce sens que cette méthode d'analyse se veut être un véritable outil méthodologique mise à la disposition des professionnels de la communication (consultants, directeurs de communication, managers, responsables des systèmes d'information et d'Internet) pour comprendre, interpréter et faire évoluer des conduites et des phénomènes communicationnels.

Cette méthode d'analyse se situe dans la lignée de l'approche compréhensive. Cette approche vise la compréhension du monde et des faits humains, tout comme les intentions des acteurs, leurs motivations, attentes, raisonnements, croyances, valeurs. (Pourtois, Desmet, 2004). La compréhension est précisément l'explicitation des significations de ces expressions, avec une volonté de conserver un critère d'objectivité se dégageant de la phase de compréhension (Minacori, 2005). Centrée sur le sens des faits humains, la volonté est de pénétrer le vécu et le ressenti de l'acteur concerné, dans un principe d'intercompréhension humaine.

En se positionnant dans une approche compréhensive, le monde décrit n'est que le reflet subjectif du point de vue des acteurs, soit une réalité co-construite par les significations portées par ces acteurs.

La sémiotique situationnelle ajoute à l'approche compréhensive six principes de lecture et d'interprétation de tout phénomène de communication :

- Le principe des définitions multiples d'une situation par différents acteurs : toute situation est délimitée et construite par l'acteur lui-même et l'acteur possède sa définition propre de la situation ; 
- le principe de la priorité de son monde vécu par l'acteur : l'acteur a une vision personnelle et partielle de la situation ;

- le principe de la construction communicationnelle et sociale de la situation partagée : les activités de communication des acteurs sociaux (conduites, attitudes, manières d'être, verbalisations) composent la situation de communication et sont la résultante des mises en relation de cette communication avec tous les éléments significatifs pour l'acteur ;

- le principe du découpage de la situation en contextes (identitaire, relationnel, normatif, de positionnement) contenant les éléments pertinents de la situation : le sens à donner à une communication dépend inéluctablement de son contexte ;

- le principe de l'accès aux données essentielles sur les situations pour les acteurs : choix des différentes techniques de recueil des données effectué par le chercheur ;

- le principe de l'accès à une vision panoramique de la situation à l'aide de la représentation graphique de l'analyse sous forme de tableau.

Cette méthode convoque généralement la triangulation de différentes techniques de recueil de données majoritairement issues des méthodes qualitatives telles que des observations simples et participantes, des entretiens, ou encore des commentaires provoqués à l'aide d'incidents critiques. Dans le cadre de l'étude présentée ci-après, nous avons choisi d'analyser les données via l'analyse de contenu thématique ${ }^{5}$. Cette technique est, quant à elle, exploitée de sorte à regrouper, à synthétiser et à analyser le corpus ensuite proposé sous forme de "mise en tableau panoramique " (Mucchielli, 2005). La volonté annoncée de la méthode est de travailler et d'affiner la représentation, et donc la modélisation de ces analyses.

La méthode d'analyse se veut véritablement une méthode constructiviste, compréhensive et qualitative ; en effet, observer la situation conduit à mettre en évidence la construction du sens émergeant en situation et dévoiler les significations pour les acteurs en présence. Sachant qu'une des principales limites apparentes se situe dans le travail interprétatif requis dans le passage des données de terrain à la catégorisation en formes contextuelles, son application suscite de nombreux questionnements. Quels sont les avantages du recours à la méthode d'analyse sémiotique situationnelle ? Quels sont les biais et écueils émergeant du travail analytique largement interprétatif ? Quels sont les intérêts du recours à cette méthode dans le champ de la communication organisationnelle ? Est-il possible d'élargir son champ d'application aux professionnels de la communication organisationnelle?

5. Pour chacune des thématiques sur lesquelles nous avons interrogé les différents groupes d'acteurs de l'organisation, nous avons réalisé une analyse de contenu qualitative, dite méthode de la catégorisation. Nous avons ainsi fait émerger le sens autour de l'ensemble de notre corpus à l'aide d'une grille de lecture permettant de confronter chacune des communications processus, de sorte à déceler des divergences, des récurrences, etc. 
Pour y réfléchir, nous fonderons notre propos sur une étude communicationnelle mettant en œuvre la méthode.

\section{II - Une étude communicationnelle mettant en œuvre la méthode dite de la sémiotique situationnelle}

Logement privilégié au cours d'une vie estudiantine, les cités universitaires hébergent chaque année des centaines d'étudiants pouvant en bénéficier ${ }^{6}$. Durant plusieurs semaines, nous avons accédé au cœur d'une cité universitaire de la ville de Montpellier de sorte à comprendre et à analyser son fonctionnement, et ainsi proposer des préconisations d'intervention auprès des acteurs de l'organisation ${ }^{7}$. En effet, les différents acteurs et groupes d'acteurs ne parvenaient pas, malgré leurs échanges, à l'intercompréhension ; le dialogue commençait à se rompre et chacun conservait des définitions divergentes et conflictuelles de la situation.

Il s'est agi d'appréhender l'organisation dans sa globalité, mais aussi et surtout dans sa complexité, en tentant de comprendre les mécanismes régissant son fonctionnement : pourquoi, malgré les insatisfactions de nombreux acteurs, la situation n'évolue-t-elle pas ? Pourquoi, malgré certains dysfonctionnements dénoncés et observés ${ }^{8}$, le système d'organisation de la cité universitaire n'est-t-il pas remis en cause ? Qu'est-ce qui poussent les acteurs (les membres du personnel de la cité universitaire) à garder le système en l'état ? Quels sont les avantages qu'ils en retirent?

Pour répondre à ces questions, nous avons mené cette recherche en adoptant une position épistémologique issue d'une " convention constructiviste " (Le Moigne, 2003). Nous avons opté pour une approche compréhensive à visée exploratoire. Notre réflexion a été guidée par triangulation méthodologique. Nous avons convoqué quatre techniques de recueil des données : les observations, les entretiens, la tenue d'un journal de bord et la technique des commentaires provoqués à l'aide d'incidents critiques.

Le recoupement de ces diverses techniques a permis de confronter les propos tenus par les acteurs en présence et les actions effectives observées sur le terrain. Nous avons fait émerger le sens à l'aide d'une analyse de contenu qualitative par catégorisation, réalisant de constants allers-retours entre les données empiriques et leur analyse. Il s'est agi d'un travail analytique largement lié aux interprétations proposées par le chercheur : «C'est un travail d'interprétation, puisqu'il s'agit de lier des conduites explicites à des principes supposés être à la source de ces conduites observées. Le travail d'induction

6. L'étude des revenus du foyer de l'étudiant est une condition sine qua non à l'accès aux logements universitaires.

7. Nous avons choisi de nous centrer uniquement sur le personnel de la cité universitaire, sans prendre en compte les étudiants hébergés

8. Les acteurs ont rapidement dénoncé des carences liées à la diffusion des informations, une mauvaise répartition des tâches, un contrôle lacunaire du travail réalisé, une mauvaise gestion du personnel de la part de certains responsables hiérarchiques, etc. 
se répète sur les enjeux des acteurs, leurs positionnements et la qualité des relations qui les lient. A chaque fois, il faut faire des hypothèses et «induire» à partir des données observables. » (Mucchielli, 2004, p. 49).

Cette méthode compréhensive est donc constamment ancrée dans les données empiriques de terrain, s'appuyant sur les expériences des acteurs, leurs propos tenus ainsi que les observations faites auprès d'eux. Elle est ainsi également itérative dans la mesure où elle demande de constats allers-retours entre les données empiriques du terrain et l'analyse de ces données. Ce travail itératif justifie également les multiples retours sur le terrain et les entretiens successifs réalisés, de sorte à recueillir des données nouvelles, voire des données plus précises. Ainsi, les phases de compréhension (des données recueillies), mise en relation (confrontation de ces données entre elles), contextualisation (mise en tableau panoramique) et interprétation (émergence du sens) sont particulièrement liées les unes aux autres et s'entrecroisent.

L'analyse communicationnelle de l'organisation s'effectue à travers des représentations graphiques (un tableau panoramique par acteur ou par groupe d'acteurs), dont nous proposons un exemple ci-dessous, soit le tableau panoramique de la situation centré sur le directeur de la cité universitaire.

Le remplissage des tableaux panoramiques pour chacun des différents acteurs en présence dans l'organisation souligne que le contexte de la qualité des relations est révélateur de sens : deux grandes catégories de relations sont présentes simultanément, les relations de mise à distance et les relations de rapprochement. Nous comprenons alors la situation totale comme révélatrice d'un jeu de l'évitement de la responsabilité et de l'engagement. Un système d'accusation réciproque justifie la logique d'insatisfaction générale ; les acteurs expliquent les dysfonctionnements de l'organisation par les erreurs des autres. En effet, chaque acteur détourne l'attention sur un autre acteur de sorte à masquer ses propres incompétences et à " garder la face " (Goffman, 1974).

Alors que la logique d'application et les apports de la méthode de la sémiotique situationnelle semblent explicites, nous souhaitons désormais nous interroger sur les limites et les usages possibles dans les recherches sur les communications organisationnelles. 


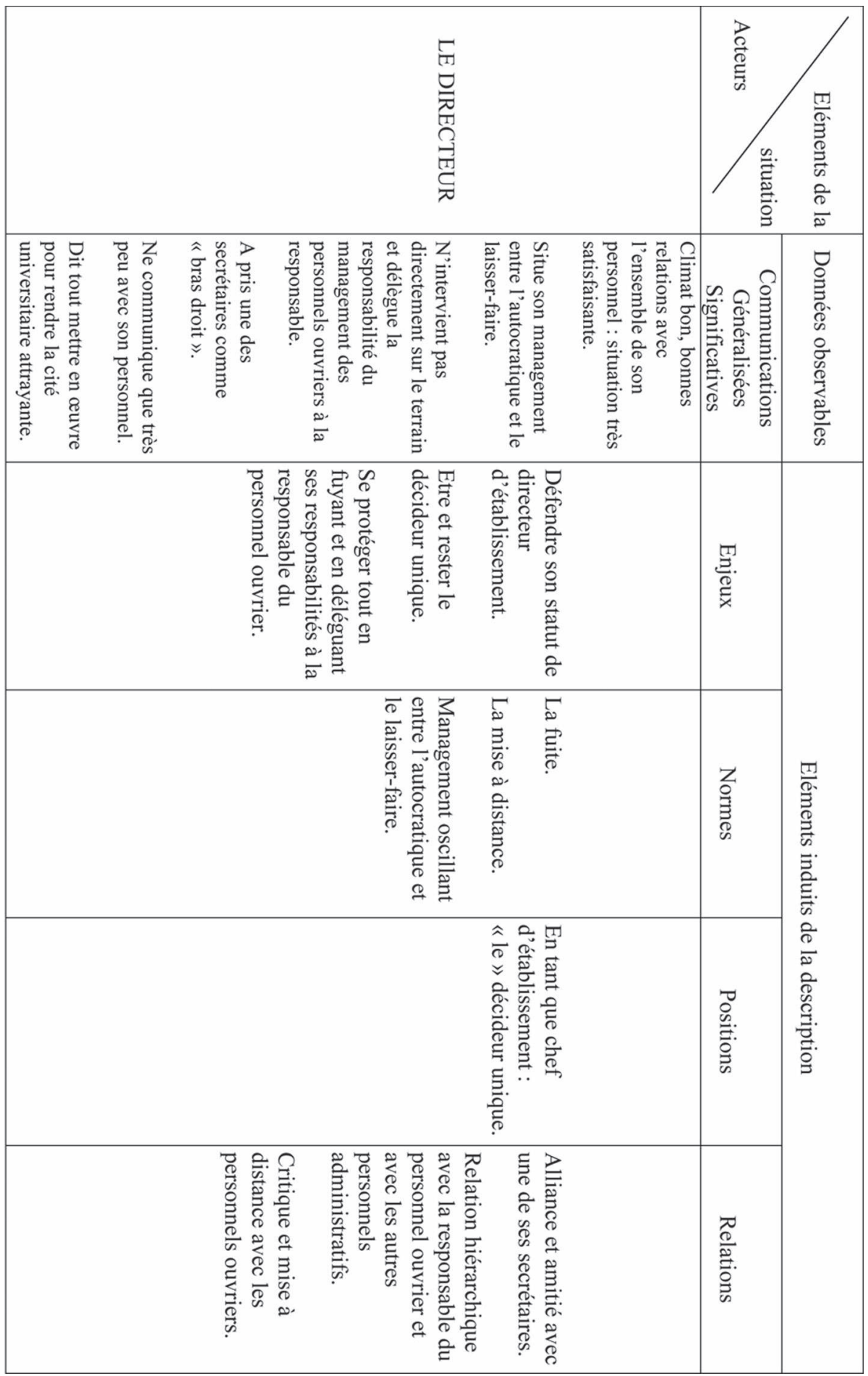




\section{III - Limites et usages dans les recherches sur les communications organisationnelles}

Le tableau panoramique de la situation se remplit en deux temps : dans un premier temps, le chercheur doit retranscrire les données observables, soit les communications généralisées significatives de l'acteur (ou du groupe d'acteurs) recueillies sur le terrain (à l'aide des différentes techniques de recueil des données choisies) ; il y a alors confrontation entre les propos tenus par les acteurs et leurs actions effectives. Dans un second temps, le chercheur doit induire de cette description et transformer ces informations en éléments porteurs de sens en termes de contexte identitaire, normatif, de positionnement et de qualité des relations.

Cette opération intellectuelle, appelée par Alex Mucchielli « catégorisation en analyse de contenu qualitative » (Mucchielli, 2005), est la résultante d'un travail d'induction et d'interprétation du chercheur qui créé le sens en faisant émerger les contextes n'existant pas a priori. C'est à ce stade de l'analyse que les risques liés à la « sur-interprétation » interviennent : «Car, la sur-interprétation guette toujours le chercheur que ce soit à partir de la réduction des cas observés, des facteurs pris en compte pour expliquer ou comprendre les faits, soit dans une recherche quasi obsessionnelle de la cohérence, si ce n'est de la généralisation abusive. ». (Héas, Poutrain, 2003, p. 13).

L'une des principales limites de la méthode réside donc autour de cette question de l'objectivation des données, tant dans la phase de recueil que dans celle de l'analyse. Le chercheur n'est pas un observateur neutre dans la mesure où il se présente sur son terrain avec des référents épistémologiques, théoriques, ou encore méthodologiques. Les phases de recueil et d'analyse des données sont ainsi entièrement liées aux référents scientifiques du chercheur, qui de ce fait ne peut être totalement objectif face à sa recherche. "Chacun, en fonction de ses expériences, de son statut social, etc., attribue des significations différentes à un même message (linguistique ou non). » (Dacheux, 2004, p. 15). De la même manière, le passage aux significations reste assez soumis aux interprétations du chercheur. En effet, le chercheur réalise un tri de façon à ne prendre en considération que les éléments lui semblant avoir de l'importance; nous pouvons une nouvelle fois mettre ici en exergue l'absence d'objectivité ainsi que la limite dans le choix de ces éléments liés principalement à l'interprétation et à la schématisation (Szafrajzen, 2010).

Toutefois, l'adoption d'une telle méthode pour l'étude des communications organisationnelles présente différents intérêts. Rappelons à ce titre que cette méthode d'analyse propose aux professionnels de la communication, quel que soit leur domaine d'action, de comprendre, d'interpréter, mais également de prévoir des conduites et des phénomènes communicationnels dans leur organisation. En ayant recours à cet outil méthodologique, le communicant peut comprendre ce qui mène à l'incompréhension (contextes opposés ou non partagés par les différents acteurs en présence dans la situation) et intervenir 
sur la situation ; le communicant permet alors d'effectuer un audit, soit une évaluation qualitative de la situation.

En outre, la méthode dite de la sémiotique situationnelle sert d'ancrage pour proposer une stratégie d'intervention ou de remédiation ; en mobilisant et en mettant en exergue un contexte comme ressource communicationnelle (plutôt qu'un autre), l'acteur impliqué dans la situation peut alors porter un regard différent sur celle-ci.

De nombreuses communications, de nombreux articles, et plus récemment des manuels (Mucchielli, 2010), proposent d'offrir aux spécialistes de la consultance et de l'intervention différentes méthodes pour comprendre et prévoir les significations attachées aux expressions et aux diverses activités humaines. Parmi ces méthodes, nous avons présenté la méthode dite de la sémiotique situationnelle, mais nous aurions également pu exposer la méthode dite de la systémique qualitative. Dans la lignée des travaux d'Alex Mucchielli, cette méthode enrichit les préceptes de l'école de Palo alto en modélisant les échanges signifiants entre les différents acteurs en présence dans la situation, sans hypothèses a priori. Les modélisations graphiques mettent en exergue la compréhension des formes et des significations des échanges au sein des systèmes étudiés. Elles sont ensuite complétées par un commentaire analytique reprenant les causalités circulaires, la logique du système (règles implicites et latentes dirigeant les acteurs) et les scénarios répétitifs des acteurs en présence. Pour chacune de ces méthodes, il s'agit d'expliquer le fonctionnement et de prévoir les règles de l'interprétation en donnant des éléments permettant d'accéder aux significations attachées aux évènements de la vie sociale.

La méthode de la sémiotique situationnelle se propose ainsi de résoudre des problématiques organisationnelles rencontrées par les professionnels de la communication dans le cadre de leurs missions. De plus en plus considérée comme un phénomène complexe, la communication organisationnelle appréhende désormais la signification des messages telle une construction en situation dépendant des contextes, mais également du processus lui-même, dans le temps et dans l'espace : il s'agit de mettre en évidence la performance des individus en situation (Bouzon, 2010).

Nous pouvons donc affirmer que la méthode présentée, tout en répondant à des critères de scientificité, est susceptible de répondre à certains besoins des professionnels de la communication, notamment dans le domaine de la consultance. En effet, la méthode peut notamment être exploitée lors d'études communicationnelles dans les organisations, de type audit, comme nous avons choisi de le faire dans le cadre de la présente étude. La méthode offre ainsi l'opportunité d'avoir une vision globalisante de l'ensemble des points de vue des acteurs en présence dans la situation. C'est en présentant cette vision panoramique regroupant l'ensemble des différentes visions de tous les acteurs qu'il est alors possible de trouver des solutions pour parvenir 
à une vision commune, connue et partagée par tous (Szafrajzen, 2010). La représentation graphique, sous forme de mise en tableaux panoramiques, de la compréhension de la situation permet de proposer aux acteurs concernés différentes significations partagées d'actions et d'évènements communs de sorte à éviter une vision étroite des phénomènes observés.

Les préconisations d'intervention proposées par le chercheur se situent dans une approche constructiviste très actuelle de l'organisation perçue comme un ensemble complexe et semi autonome de relations prenant leur origine dans les interactions humaines (Bouzon, 2010).

\section{Conclusion}

La méthode dite de la sémiotique situationnelle, appliquée à une recherche en communication des organisations, présente différents apports et limites que nous avons succinctement évoqués dans le présent article. Cette présentation de certains des résultats de cette recherche à visée exploratoire ne prétend pas à l'exhaustivité mais tente plus modestement de témoigner d'une expérience récente et d'enrichir la réflexion méthodologique pour le champ de la communication des organisations.

Le recours au terme "sémiotique » est justifié par l'idée d'émergence du sens en situation et en interaction avec son contexte d'émergence. La genèse du sens partagé est la problématique au cœur de cette méthode de recherche et d'intervention. Permettant de s'éloigner des représentations cloisonnées qu'ont les acteurs de leur situation, la méthode offre alors une vision nouvelle d'une situation bien plus globale et bien plus complexe.

Se voulant à la fois constructiviste, compréhensive et qualitative, les spécialistes de la communication pressentent les intérêts du recours à cette méthode dans le champ de la communication organisationnelle. La méthode propose en effet de comprendre et de prévoir les significations attachées aux évènements de la vie sociale. À ce titre, cette méthode se veut un outil pragmatique très concret liant étroitement chercheurs et praticiens, notamment dans le domaine de la consultance et de l'intervention.

\section{BIBLIOGRAPHIE}

BOUTAUD J.-J., VERON E., Sémiotique ouverte, Itinéraires sémiotiques en communication, Paris-Londres, Lavoisier-Hermès Science Publishing, «Forme et sens », 2007, $192 \mathrm{p}$.

BOUZON A., «Comunicación y organización. Orígenes y fundamentos de los trabajos científicos francófonos », In II Congreso Internacional de la Asociación Española de Investigadores de la Comunicación (AE-IC), Comunicación y desarrollo en la era digital, 3 
à 5 de febrero de 2010, Facultad de Ciencas de la Comunicación de la Universidad de Málaga, 2010, 22 p.

DACHEUX E., "La communication : éléments de synthèse », Communication et langages, $\mathrm{n}^{\circ} 141,2004,29 \mathrm{p}$.

GOFFMAN E., Les rites d'interaction, Paris, Les Éd. de Minuit, 1974, 230 p.

HÉAS S., POUTRAIN V., " Les méthodes d'enquête qualitative sur Internet. In revue en ligne de sciences humaines et sociales ", Ethnographiques.org, [en ligne], $\mathrm{n}^{\circ}$ 4, novembre 2003 [consulté le 15 décembre 2006]. Disponible sur : http://www. ethnographiques.org/2003/Heas,Poutrain.html

LELEU-MERVIEL S., "Effets de la numérisation et de la mise en réseau sur le concept de document ", I3: Information, Interaction, Intelligence, A Journal in the Sciences of Information Engineering, 2004, vol. 4, n 1, Paris, Cépaduès Editions, p. 121-140.

LE MOIGNE J.-L., Le constructivisme. Modéliser pour comprendre. Tome 3, Paris, L'Harmattan. 2003, 335 p.

MUCCHIELLI A., Dictionnaire des méthodes qualitatives en sciences humaines. $2^{\mathrm{e}}$ édition, Paris, Armand Colin, 2004, 304 p.

MUCCHIELLI A., Étude des communications : approche par la contextualisation, Paris, Armand Colin, 2005, 264 p.

MUCCHIELLI A., La méthode du tableau panoramique pour l'étude des situations, CERIC, Montpellier 3, Février 2003, 23 p.

SCHÜTZ A., Essais sur le monde ordinaire (trad., T. Blin), Paris, Félin Poche, 2007, 202 p.

SZAFRAJZEN B., Lecture communicationnelle de deux dispositifs d'apprentissage au sein d'un même département universitaire. Le cas de la licence en sciences de l'information et de la communication. Thèse de doctorat en Sciences de l'information et de la communication. Université Montpellier III, Montpellier, France, 2010, 445 p.

Résumé : La communication organisationnelle est un des domaines des sciences de l'information et de la communication à se nourrir des recherches en sémiotique-sémiologie. Parmi les méthodologies possibles de cette relation, Alex Mucchielli propose la méthode d'analyse dite de la sémiotique situationnelle au début des années 2000 (Mucchielli,2003). Cet article propose différentes réflexions questionnant et exposant les biais et limites de la méthode, en se fondant sur un corpus issu d'une étude communicationnelle réalisée au sein d'une cité universitaire de la ville de Montpellier (Hérault, France). En mettant en perspective les résultats obtenus, il s'agit de tenter de contribuer à une réflexion mettant en tension les champs de la sémiotique à la communication des organisations.

Mots-clefs : Méthode, sémiotique situationnelle, contextualisation, situation, signification.

Abstract : The organizational communication is one among other domains in the field of information and communication sciences fed by researches in semiotics-semiology. Among other possible forms of this relation, Alex Mucchielli proposed the method of situational semiotics 
(sémiotique situationnelle) at the beginning of 2000s (Mucchielli, on 2003). This article proposes various reflections questioning and exposing the ways and limits of the method, by establishing itself on a corpus stemming from a communicational study realized within a student hall of residence in the city of Montpelier (the Hérault, France). By putting in prospect the obtained results, it is a question of trying to contribute to a reflection putting in tension the fields of semiotics and the communication of organizations.

Keywords : method, situational semiotics, contextualization, situation, meaning. 